\title{
Improvement of Wearable Wrist Rehabilitation Device Using Flexible Pneumatic Cylinders
}

\author{
Naoki Kato, Shujiro Dohta, Tetsuya Akagi ${ }^{\text {a }}$, Wataru Kobayashi and Mohd Aliff \\ Department of Intelligent Mechanical Engineering, Okayama University of Science, \\ 1-1, Ridai-cho, Kita-ku, Okayama, 700-0005, Japan
}

\begin{abstract}
In the condition of the insufficient number of PT, the novel wearable rehabilitation devices using a soft pneumatic actuator for the elderly are actively proposed and developed. In the previous study, a wearable wrist rehabilitation device based on a flexible robot arm using three flexible pneumatic cylinders was proposed and tested. In this study, the improvement of the device is carried out so as to use it without limitation of attitude of patient's arm. The attitude control of the improved device based on the analytical model is also carried out. As a result, it is confirmed that the device can give the motion to patients with any attitude of their arm according to the desired position.
\end{abstract}

\section{Introduction}

Based on the situation of aging society and decreasing birth rate[1], it becomes serious concern that the number of welfare workers and physical therapists ("PT" for short) is not enough. The development of rehabilitation devices using soft pneumatic actuators for the disabled and elderly has been carried out actively [2-5]. In the previous study, the wrist rehabilitation device based on the flexible robot arm using three flexible pneumatic cylinders [6] was proposed and tested. The attitude control of the robot arm while the patient is holding the upper stage in the robot arm was executed [7] [8]. The robot arm that has a function of simple back-drivability was also proposed and tested [9]. In the therapy using the device, the patient's hands must be put on the robot on the table. Therefore, the wrist rehabilitation device that a patient worn it was also proposed and tested [10]. In the next step, in order to use it while the device is not fixed on the table, the improvement of attitude control system of the device is required. In this study, the improvement of attitude control system of the device using an embedded controller and two accelerometers is investigated. The flexible pneumatic cylinder is a kind of new soft actuator. This actuator has an advantage of flexibility based on the air compressibility and the usage of flexible tube. We believe that the flexible cylinder will contribute to extend the robotics application fields as proposed in this paper.

\section{Flexible pneumatic cylinder and wearable wrist rehabilitation device}

a Corresponding author : akagi@are.ous.ac.jp 
The construction and movement of the flexible pneumatic cylinder which is a basic component in the proposed rehabilitation device is shown in Fig.1. The cylinder consists of these main parts; a flexible tube as a cylinder, the steel ball as a cylinder head, and a slide stage. The slide stage has two rollers set on the inner bore of the stage to press and deform the tube. The steel ball is held by two slide stages from both sides of the ball. When the pressure is applied to one side of the cylinder, the tube is moved forward or backward while holding the slide stage. This is a kind of rodless-type cylinder. The slide stage can move smoothly on the curved cylinder tube.
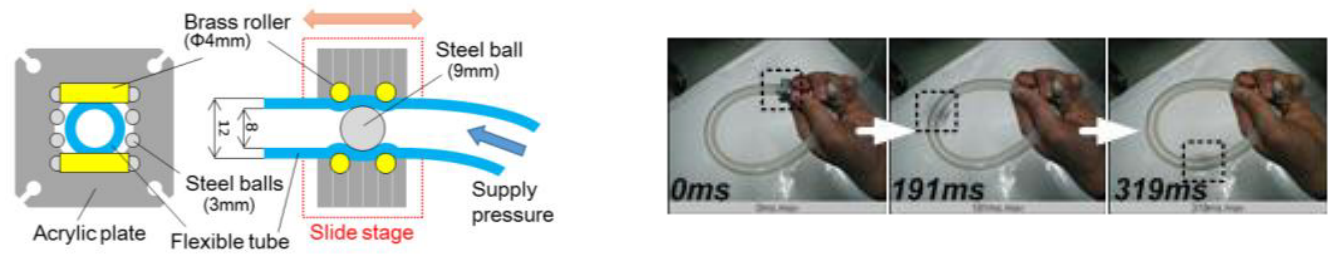

Figure 1. Construction and movement of flexible pneumatic cylinder.

Figure 2 shows the construction of the tested wearable type wrist rehabilitation device using flexible pneumatic cylinders that we had developed [11]. The device consists of three flexible pneumatic cylinders and two round stages. Each flexible pneumatic cylinder is arranged on the round stage with radius of $87.5 \mathrm{~mm}$ every $120 \mathrm{deg}$. from the center. An end of each flexible cylinder is fixed in the base stage. The end stage has a hole with the inner diameter of $100 \mathrm{~mm}$ so that a human arm can insert to it. On the end stage that has a handle, the slide stage of each cylinder is fixed. In rehabilitation, the patient arm passes through the holes of the base stage. The patient also holds the handle while working. In order to realize a wearable and portable device, the attitude control system that consists of an embedded controller (Renesas Co. Ltd., SH7125) and six quasi-servo valves[12] are mounted on the device. The quasi-servo valve consists of two on/off type valves. One is a switching valve for supply or exhaust and the other is PWM controlled valve for adjusting flow rate. The embedded controller and three quasi-servo valves are mounted on the base stage. On the end stage, there are three quasi-servo valves and an accelerometer for measuring the inclined angle of the stage. In order to supply the compressed air from the base stage to the end stage, a coil type tube that covers on a flexible pneumatic cylinder was used. By this method, the device requires only an electric power cable and an air supply pipe to drive it. The device has the outer diameter of $200 \mathrm{~mm}$ and the length of 420 $\mathrm{mm}$. The total mass of the device including a controller and six quasi-servo valves is $1.14 \mathrm{~kg}$.
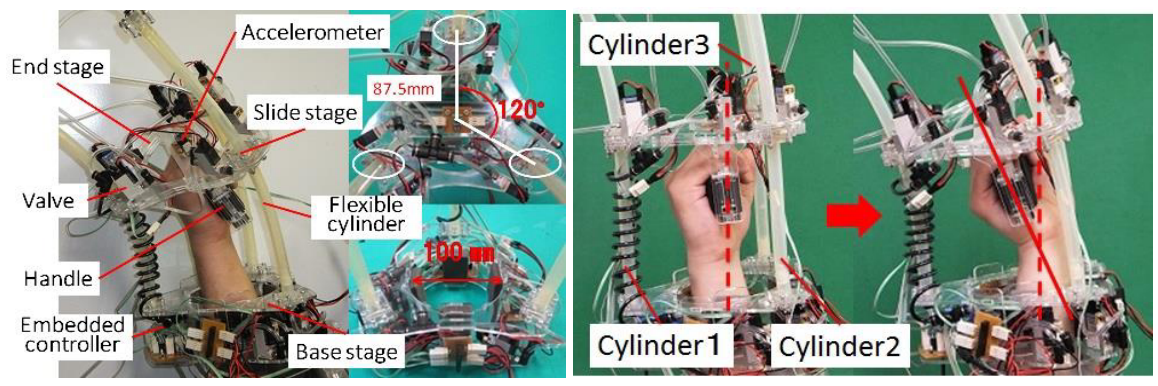

Figure 2. Construction of wearable wrist rehabilitation device using flexible pneumatic cylinders.

\section{Attitude control system of tested device}

Figure 3 shows the schematic diagram of the control system of the previous wrist rehabilitation device. The system consists of the flexible robot arm using three flexible pneumatic cylinders, an accelerometer, the embedded controller (Renesas Co. Ltd., SH7125), and six quasi-servo valves. In order to execute the attitude control of the device, it is necessary to measure the displacement of the 
flexible pneumatic cylinder. However, it is difficult to measure the displacement of the cylinder directly because of its flexibility. Therefore, indirect measurement based on the theoretical model of the arm was carried out.

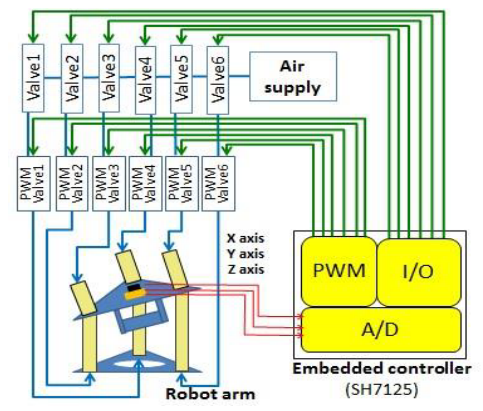

Figure 3. Schematic diagram of control system.

Figure 4 shows the definition of the cylinder length and the analytical model of the wearable wrist rehabilitation device for the control. In this model, the shape of the flexible pneumatic cylinder is assumed to be a circular arc when the device is bent. From the center of the rehabilitation device, the bending angle from $\mathrm{X}$ axis is called a bending direction angle $\alpha$ while the bending angle $\beta$ is defined as the angle between the normal vectors from the center of the end stage of the $\mathrm{Z}$ axis of the device. From the geometric relationship, the following equations about cylinder length $L_{1}, L_{2}, L_{3}$ and the radius of curvature $R$ can be obtained.

$$
\begin{aligned}
& L_{1}=(R-r \cdot \cos \alpha) \cdot \beta \\
& L_{2}=\left\{R-r \cdot \cos \left(\frac{2 \pi}{3}-\alpha\right)\right\} \cdot \beta \\
& L_{3}=\left\{R-r \cdot \cos \left(\frac{4 \pi}{3}-\alpha\right)\right\} \cdot \beta \\
& R=L / \beta
\end{aligned}
$$

where, $L$ means the length of human arm, and $r=87.5 \mathrm{~mm}$ is the distance from the center to the cylinder. Angles $\alpha$ and $\beta$ can be obtained from the output voltage of the accelerometer.
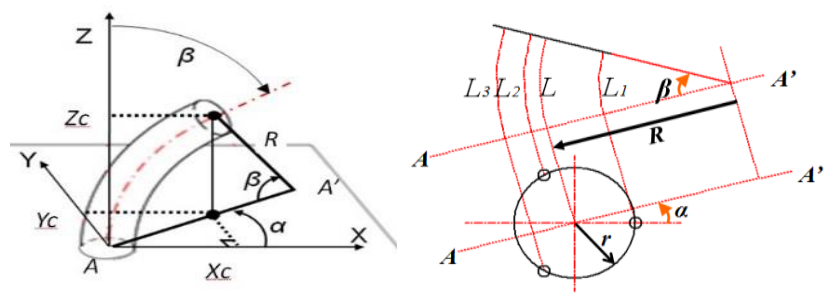

Figure 4. Analytical model of wearable device (flexible robot arm).

The attitude control of the device using the analytical model is done as follows. The embedded controller gets the output voltages from the accelerometer through $\mathrm{A} / \mathrm{D}$ converter and angles $\alpha$ and $\beta$ are calculated. Then, each length of the flexible pneumatic cylinder is calculated by Eqs.(1) to (4).

And, the embedded controller also calculates the deviation from the desired position for each cylinder. Finally, the quasi-servo valves are driven according to the control scheme. The desired position is set on the embedded controller in advance. 


\section{Attitude control result of the device}

Figure 5 shows the transient response of each cylinder length in multi-position control. In the control, the following PID control scheme with sampling period of $7 \mathrm{~ms}$ was used.

$$
\begin{aligned}
& u_{i(j)}=K p \cdot d_{i(j)}+K d \cdot\left(d_{i(j)}-d_{i(j-1)}\right)+K i \cdot \sum d_{i(j)} \\
& \text { Switching valves } \\
& \text { End stage : OFF, Base stage : ON } \quad\left(u_{i(j)}>0\right) \\
& \text { End stage : ON, Base stage : OFF } \quad\left(u_{i(j)}<0\right) \\
& \text { End stage : OFF, Base stage : OFF } \quad\left(u_{i(j)}=0\right)
\end{aligned}
$$

where, $d_{i(j)}$ and $u_{i(j)}$ mean the current deviation of cylinder displacement and the differential input duty ratio for PWM valves, respectively. As an input duty ratio, $22.5 \%$ is added to compensate the dead zone of PWM valves[11]. $i$ shows the cylinder number 1,2, and 3. The control parameters of $K p=$ $3.5 \% / \mathrm{mm}, K d=2.5 \% / \mathrm{mm}$, and $K i=0.05 \% / \mathrm{mm}$ were decided by trial and error.

In the experiment, the initial length $L$ was set to be $150 \mathrm{~mm}$. In Fig.5, the broken and solid lines show the desired and controlled length of each cylinder, respectively. It can be seen that the cylinder can trace the desired length well. For changing desired position, it reaches at the desired position within approximately one second even if the force from the human wrist is applied to the device as a load.
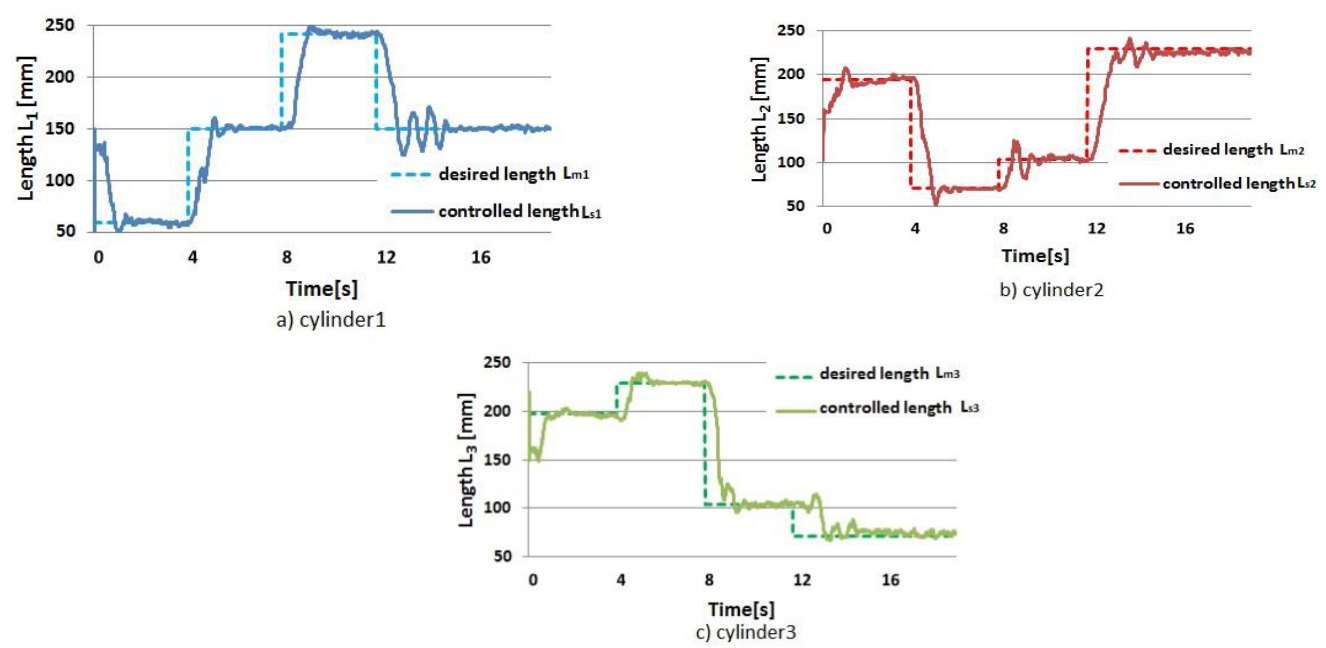

Figure 5. Transient response of cylinder length in multi-position control.

\section{Improvement of wearable wrist rehabilitation device}

Figure 6 shows the schematic diagram of the control system of the improved wearable wrist rehabilitation device. The fundamental construction of control system is similar to the device. Compared with the previous system, an accelerometer is added and set on the base stage. The attitude control of the device is carried out as follows. The embedded controller gets the output voltages from two accelerometers through $\mathrm{A} / \mathrm{D}$ converter. From $\mathrm{A} / \mathrm{D}$ value of $x, y$, and $z$ direction of each accelerometer, a bending direction angle $\alpha$ and the bending angle $\beta$ of each stage can be calculated. Differential bending angles $\alpha$ and $\beta$ are given by Eq.(7) and Eq.(8), respectively. 


$$
\begin{aligned}
& \alpha=\alpha_{e}-\alpha_{b} \\
& \beta=\cos ^{-1}\left(\cos \beta_{e} \cdot \cos \beta_{b}-\sin \beta_{e} \cdot \sin \beta_{b} \cdot \cos \alpha\right)
\end{aligned}
$$

where, subscripts $e$ and $b$ mean the end and base stages, respectively. Eq.(8) can be obtained by the geometical relationship between the base stage and the end stage. When $\alpha_{e}=\alpha_{b}$, that is $\alpha=0$, the bending angle $\beta$ can be expressed by the difference $\beta_{e}-\beta_{b}$. This is obviously obtained by Eq.(8). By using the calculated $\alpha$ and $\beta$, each length of the flexible pneumatic cylinder is calculated by Eqs.(1) to (4). The embedded controller also calculates the deviation from the desired position for each cylinder. The quasi-servo valves are driven according to the $\mathrm{P}$ control scheme.

Figure 7 shows the transient response of each cylinder length in multi-position control. In the experiment, the base stage has the inclined angle of approximately $20 \mathrm{deg}$. from the horizontal plane as an initial condition. In the control, PID control scheme with sampling period of $7 \mathrm{~ms}$ and the control parameters gain of $K p=3.5 \% / \mathrm{mm}, K d=3.5 \% / \mathrm{mm}$, and $K i=0.3 \% / \mathrm{mm}$ was used. The same desired positions as the previous experiment were given. In Fig.7, the broken and solid lines show the desired and controlled length of each cylinder, respectively. It can be seen that the cylinder can trace the desired position. As a result, it can be confirmed that the device can give the motion to patient's wrist even if the patient's arm is not set parallel to the vertical plane. It can be observed that there are oscillation around the desired position. This seems to be caused by adding the accelerometer to the control system. The controlled performance will be improved by applying superior control scheme.

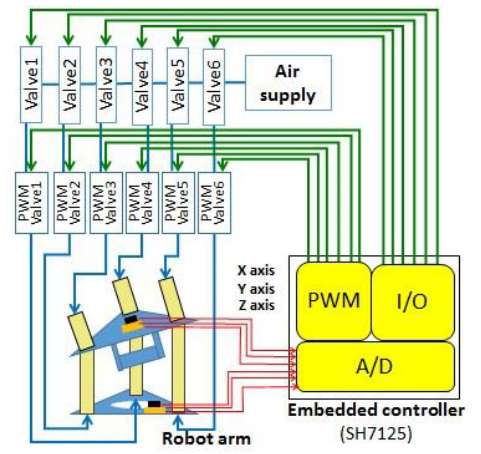

Figure 6. Schematic diagram of improved control system.
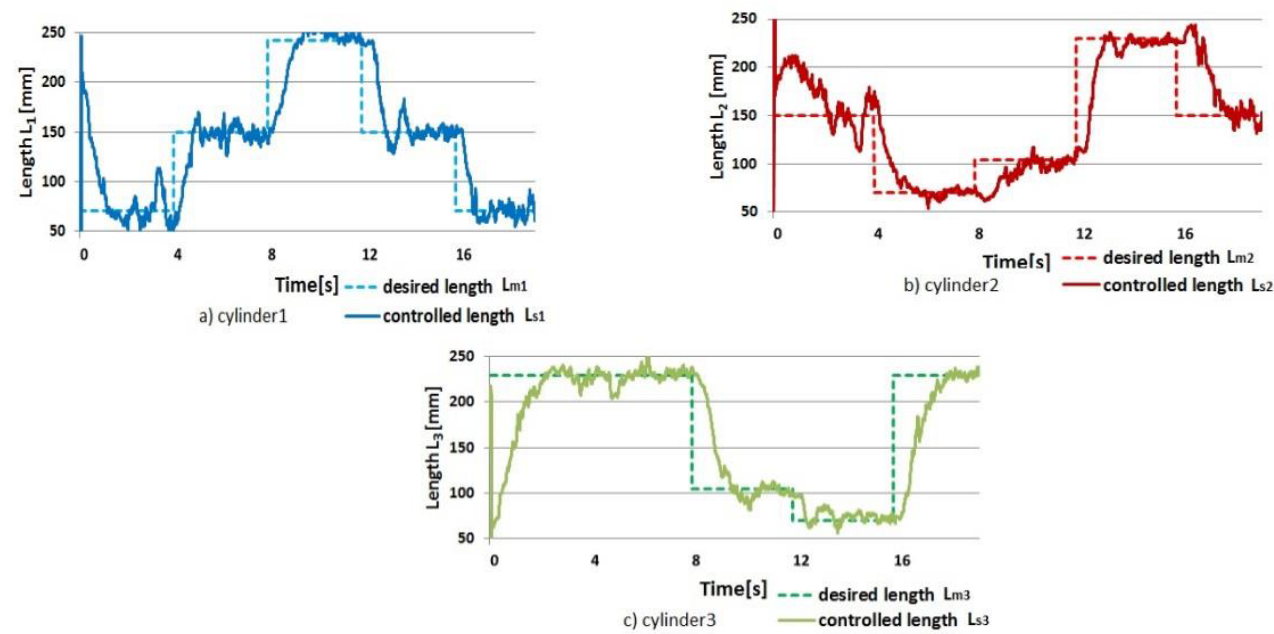

Figure 7. Transient response of cylinder length in multi-position control. 


\section{Conclusions}

The improved wearable wrist rehabilitation device using three flexible pneumatic cylinders, an embedded controller, six quasi-servo valves, and two accelerometers is proposed and tested. The measuring system of differential bending angle between the base and the end stage of the device is also constructed in the built-in embedded controller. By using this measuring system, the attitude control of the improved device can be realized. As a result, it can be confirmed that the device can give the motion to patient's wrist even if the patient's arm is not set parallel to the vertical plane.

\section{Acknowledgements}

This research was supported by the Ministry of Education, Culture, Sports, Science and Technology of Japan through a Financial Assistance Program for QOL Innovative Research (2012-2016).

\section{References}

1. Ministry of Internal Affairs and Communications, Statistics Bureau, Statistics, Population Estimates, Result of the Population Estimates, Monthly Report. Result of the Population Estimates (online), Available: http://www.stat.go.jp/english/data/jinsui/tsuki/ (2015)

2. M. Ishii, K. Yamamoto, K. Hyodo. Stand-Alone Wearable Power Assist Suit -Development and Availability-, Journal of Robotics and Mechatronics, Vol.17, No.5, 575-583 (2005)

3. J. Piquion, A. Nayar, A. Ghazaryan, R. Papann, W. Klimek, and R. Laroia, Robot-assisted gynecological surgery in a community setting, Journal of Robotics and Surgery, Vol.3, Issue 2, 61-64 (2009)

4. T. Noritsugu, M. Takaiwa, D. Sasaki, Development of Power Assist Wear Using Pneumatic Rubber Artificial Muscles, Journal of Robotics and Mechatronics, Vol. 21, No. 5, 607-613 (2009)

5. H. Kobayashi, T. Shiban, Y. Ishida, Realization of all 7 motions for the upper limb by a muscle suit, Journal of Robotics and Mechatronics, Vol. 16, 504-512 (2004)

6. T. Akagi, S. Dohta, Development of a Rodless Type Flexible Pneumatic Cylinder and Its Application, Transactions on Robotics and Automation of the JSME (C), Vol. 73, No. 731, 21082114 (2007) (in Japanese)

7. T. Akagi, S. Dohta, Feifei Zhao, T. Fujikawa, Development and Attitude Control of Flexible Robot Arm Using Flexible Pneumatic Cylinder with Simple Structure, International Journal of Automation Technology, Vol.5, No.4, 523-530 (2011)

8. M. Aiff, T. Akagi, S. Dohta, Control and Analysis of Robot Arm Using Flexible Pneumatic Cylinder, Mechanical Engineering Journal, Vol.1, No.5, 1-13 (2014)

9. T. Morimoto, T. Akagi, S. Dohta, Development of Flexible Haptic Robot Arm Using Flexible Pneumatic Cylinders with Backdrivability for Bilateral Control, Lecture Notes in Electrical Engineering 345, Springer, 231-237 (2016)

10. T. Morimoto, M. Aiff, T. Akagi, S. Dohta, Development of Flexible Pneumatic Cylinder with Backdrivability and Its Application, International Journal of Materials Science and Engineering, Vol.3, No.1, 7-11 (2015)

11. N. Kato, S. Dohta, T. Akagia, T. Morimoto, Y. Soga, Development of Wearable Wrist Rehabilitation Device Using Flexible Pneumatic Cylinders, MATEC Web of Conferences, Vol.51, No.02003, 1-5 (2016)

12. F. Zhao, T. Akagi, S. Dohta, Development of Small-Sized Quasi-Servo Valve for Flexible Bending Actuator, Proc. 2009 CACS Int. Automatic Control Conference, 558-562 (2009) 